\title{
Efficacy and safety profile of paliperidone palmitate injections in the management of patients with schizophrenia: an evidence-based review
}

This article was published in the following Dove Press journal:

Neuropsychiatric Disease and Treatment

\section{Robin Emsley \\ Sanja Kilian}

Department of Psychiatry, Faculty of Medicine and Health Sciences, Stellenbosch University, Cape Town, South Africa
Correspondence: Robin Emsley Department of Psychiatry, Faculty of Medicine and Health Sciences, Stellenbosch University, PO Box 24I, Tygerberg Campus 8000, Cape Town, South Africa

Tel +27 21 9389227

Email rae@sun.ac.za

\begin{abstract}
The course of schizophrenia is characterized by multiple relapses, incomplete remission of symptoms, enduring cognitive deficits, and social and occupational functional impairments. Nonadherence to antipsychotic medication is a major determinant of this poor outcome. Long-acting injectable antipsychotics were developed specifically to address the nonadherence problem and are increasingly considered as an early treatment option, in an attempt to prevent accruing morbidity. This review focuses on paliperidone palmitate, the long-acting injectable (LAI) formulation of paliperidone. After considering the pharmacology of paliperidone palmitate, we review the randomized controlled trials, as well as pertinent observational, pragmatic studies for paliperidone once-monthly injections in schizophrenia. Finally, we review the recently introduced 3-monthly formulation of paliperidone palmitate. Taken together, the studies indicate that paliperidone palmitate (PP) has good efficacy compared with placebo and comparable with other antipsychotics including risperidone. The tolerability profile of PP is similar to that of risperidone, with the most important side effects being prolactin elevation, weight gain, and extrapyramidal symptoms. Advantages of PP include the extensive research database and clinical experience with paliperidone and its parent compound risperidone, the availability of different LAI formulations (once-monthly, 3-monthly, and perhaps even longer acting formulations in future), and the novel dose initiation procedure that provides rapid onset of action without the need for oral antipsychotic supplementation.
\end{abstract}

Keywords: paliperidone palmitate, long-acting antipsychotics, schizophrenia, relapse-prevention

\section{Introduction}

As a chronic and frequently debilitating brain disorder, schizophrenia is one of the leading contributors to the global disease burden. Despite its relatively low prevalence, the illness places an enormous emotional, health, social, and economic burden on patients, families, caregivers, and society. ${ }^{1}$ The course of schizophrenia is typically characterized by multiple relapses, incomplete remission of symptoms, enduring cognitive deficits, and social and occupational functional impairments. ${ }^{2}$ There is also evidence for emergent treatment refractoriness, with relapse events possibly being the critical factor. ${ }^{3}$ Antipsychotic treatment is effective in reducing acute psychotic symptoms and for preventing relapse by way of maintenance treatment. Despite the demonstrated effectiveness of antipsychotics, the need for indefinite treatment has recently been questioned, due to the side effect burden of these drugs and a study suggesting that patients who undergo dose reduction or discontinuation may do better in the long term in terms of functional recovery. ${ }^{4}$ However, the effectiveness of maintenance antipsychotic treatment is extensively documented. ${ }^{5}$ Relapse rates are 
very high when treatment is discontinued, even after a single episode of psychosis, ${ }^{6}$ and good adherence and persistence with antipsychotics is associated with reduced hospitalization risk and lower total costs. ${ }^{7}$ Given the psychosocial and possible biological risks associated with discontinuation, ${ }^{8}$ most clinicians continue to encourage indefinite maintenance treatment.

While it is established that schizophrenia is a neurodevelopmental disorder, ${ }^{9}$ there is also evidence of illness progression after onset of the first psychotic episode. The illness trajectory has been proposed as having two phases. The first phase, comprising the early years, is when the illness is at its most aggressive, when relapses are most common, and where illness progression is most likely to occur. The second, chronic phase is when the illness has burnt itself out and patients stabilize insofar as acute psychotic episodes are less severe, although prominent deficits persist. ${ }^{10}$ Indeed, long-term data indicate that, even in patients who become relatively symptom free, psychosocial deficits are prominent and few achieve self-sufficiency and independent living. ${ }^{11}$

The importance of early identification, effective intervention, and prevention of relapse in the early years of illness has been emphasized as the best strategy to minimize illness progression. First-episode patients are more responsive to acute treatment, ${ }^{12}$ although frequently the improvement is not maintained. This is consistent with a longitudinal study in patients with schizophrenia treated with oral antipsychotics where it was reported that, while $70 \%$ of participants met cross-sectional criteria for symptom remission at some time point in the study, only $23.6 \%$ maintained that status for 6 months or longer. ${ }^{13}$ This suggests that, in the early stages of illness, the challenge is not so much in achieving a treatment response, but in maintaining that status. Perhaps the most important barrier to optimal outcomes is nonadherence to and poor persistence with treatment. About $80 \%$ of patients experience multiple relapses over the first 5 years of treatment, and antipsychotic treatment discontinuation is by far the commonest predictor of relapse. ${ }^{14}$ The very high rates of discontinuation in studies such as the CATIE trial highlight the extent of the problem. ${ }^{15}$ While nonadherence and poor persistence is common in most chronic illnesses, the problem is compounded in schizophrenia where insight is impaired to the extent that there may be illness unawareness, symptom misattribution, and failure to recognize the need for treatment. ${ }^{16}$ For these reasons, psychosocial and pharmacological interventions have focused on ways of improving adherence and persistence. Long-acting injectable antipsychotics (LAIs) were developed in the 1960 s specifically to address the nonadherence problem, and the older, first-generation depot antipsychotics were used extensively in many countries as an effective way of ensuring maintenance treatment. ${ }^{17}$ However, these agents were typically reserved for the chronic stage of illness, for patients who had proven themselves to be nonadherent to medication and after multiple relapses. In keeping with modern day public health principles, it makes sense rather to address nonadherence and poor persistence from the outset, thereby preventing accruing morbidity. ${ }^{18}$ Consequently, the use of LAIs as an early treatment option is increasingly considered. With the advent of the new generation oral antipsychotics two decades ago, it was hoped that these drugs would, by virtue of their more favorable tolerability profile, improve adherence and persistence. As a result, use of LAIs decreased. However, the newer oral antipsychotics failed to substantially improve adherence and reduce relapse rates. ${ }^{17,19}$ Subsequently, attention has again focused on developing LAI formulations, this time of the new generation antipsychotics. There are now LAI formulations for risperidone, olanzapine, aripiprazole, and paliperidone. The present selective review focuses specifically on paliperidone palmitate (PP), the LAI formulation of paliperidone. We conducted a Medline search using the search term "paliperidone palmitate". References of the identified studies were cross-checked for additional relevant studies.

\section{Pharmacology, mode of action, pharmacokinetics, and administration schedule}

Paliperidone (9-hydroxyrisperidone) is the active metabolite of risperidone. Risperidone and paliperidone have similar receptor binding profiles, although there are some differences. While paliperidone antagonizes central dopamine D2 and serotonin 5-HT2A receptors as well as alpha1- and alpha 2-adrenergic receptors and $\mathrm{H} 1$ histaminergic receptors, the risperidone 5-HT2A/D2 binding ratio is significantly lower than that of paliperidone. Also, a hydroxyl group in the paliperidone molecule confers increased hydrophilicity that contributes to differential effects on mitochondrial movement, protein expression, and phosphorylation. ${ }^{20}$ Further, paliperidone may be less able to enter the brain due to its greater affinity for the transporter P-glycoprotein. Whether these pharmacological differences result in clinical differences is not established. Renal excretion is the major route of paliperidone elimination. As such, PP is not recommended in patients with moderate or severe renal impairment. ${ }^{21}$ In vitro studies in human liver microsomes found that paliperidone does not substantially inhibit the 
metabolism of medicines metabolized by cytochrome $\mathrm{P} 450$ isozymes, including CYP1A2, CYP2A6, CYP2C8/9/10, CYP2D6, CYP2E1, CYP3A4, and CYP3A5. Therefore, in contrast to risperidone, no dose adjustment is required for patients with mild to moderate hepatic impairment, and being a CYP2D6 poor metabolizer may not be clinically relevant for paliperidone treatment. ${ }^{22}$

PP is the palmitate ester of paliperidone, provided as a nanocrystal suspension in an aqueous vehicle for parenteral administration. Due to its low water solubility, PP dissolves slowly after intramuscular injection before being hydrolyzed to paliperidone and absorbed into the systemic circulation. PP may be administered either as gluteal or as deltoid intramuscular injection. The original once-monthly injectable formulation of PP (PP-1M) was approved by the US Food and Drug Administration (FDA) in 2009 and is marketed as Invega Sustenna ${ }^{\circledR}$ (Janssen Pharmaceutica NV, Beerse, Belgium) in the United States and as Xeplion ${ }^{\circledR}$ (Janssen Pharmaceutica NV) in Europe. Mean $\mathrm{C}_{\max }$ was higher after deltoid injection than after gluteal injection (except for the $100 \mathrm{mg}$ equivalent [eq.] dose), although the area under the curve extrapolated to infinity divided by weight $\left(\mathrm{AUC}_{\text {infinity }}\right)$ for both injection sites was comparable at all doses. Median time to reach $\mathrm{C}_{\max }$ ranged from 13 to 14 days after deltoid and 13 to 17 days after gluteal injection across all doses. ${ }^{23}$

The recommended initiation of PP is with a dose of $150 \mathrm{mg}$ on treatment day 1 and $100 \mathrm{mg} 1$ week later (day 8), both administered in the deltoid muscle in order to attain rapid therapeutic concentrations. The third dose should be administered 1 month after the second initiation dose. The recommended monthly maintenance dose is $75 \mathrm{mg}$, and the recommended dose range is $25-150 \mathrm{mg}$. Patients who are overweight or obese may require doses in the upper range. Monthly maintenance doses can be administered in either the deltoid or gluteal muscle. ${ }^{21}$

\section{Randomized, controlled trials vs placebo for acute, symptomatic schizophrenia}

Efficacy and safety of flexible doses of PP-1M was demonstrated in four short-term double-blind, placebo-controlled, randomized, controlled trials (RCTs) (Table 1). In a Phase 2b study over 9 weeks, acutely symptomatic participants $(n=197)$ received gluteal injections of PP (fixed doses of 50 or $100 \mathrm{mg}$ eq.) or placebo on days 1,8 , and 36. Positive and Negative Syndrome Scale (PANSS) total scores improved significantly at end point for both doses $(P<0.01)$ vs placebo. Fewer PP-treated (2\%) vs placebo-treated (10\%) patients discontinued the study due to emergent adverse

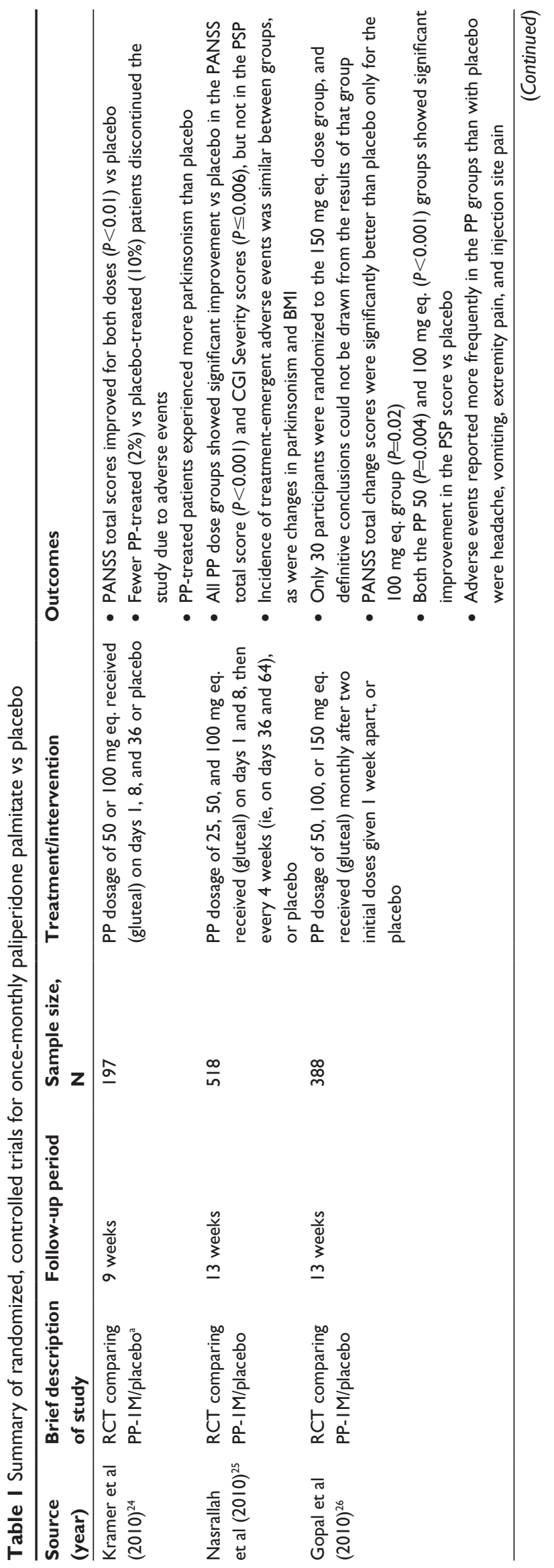




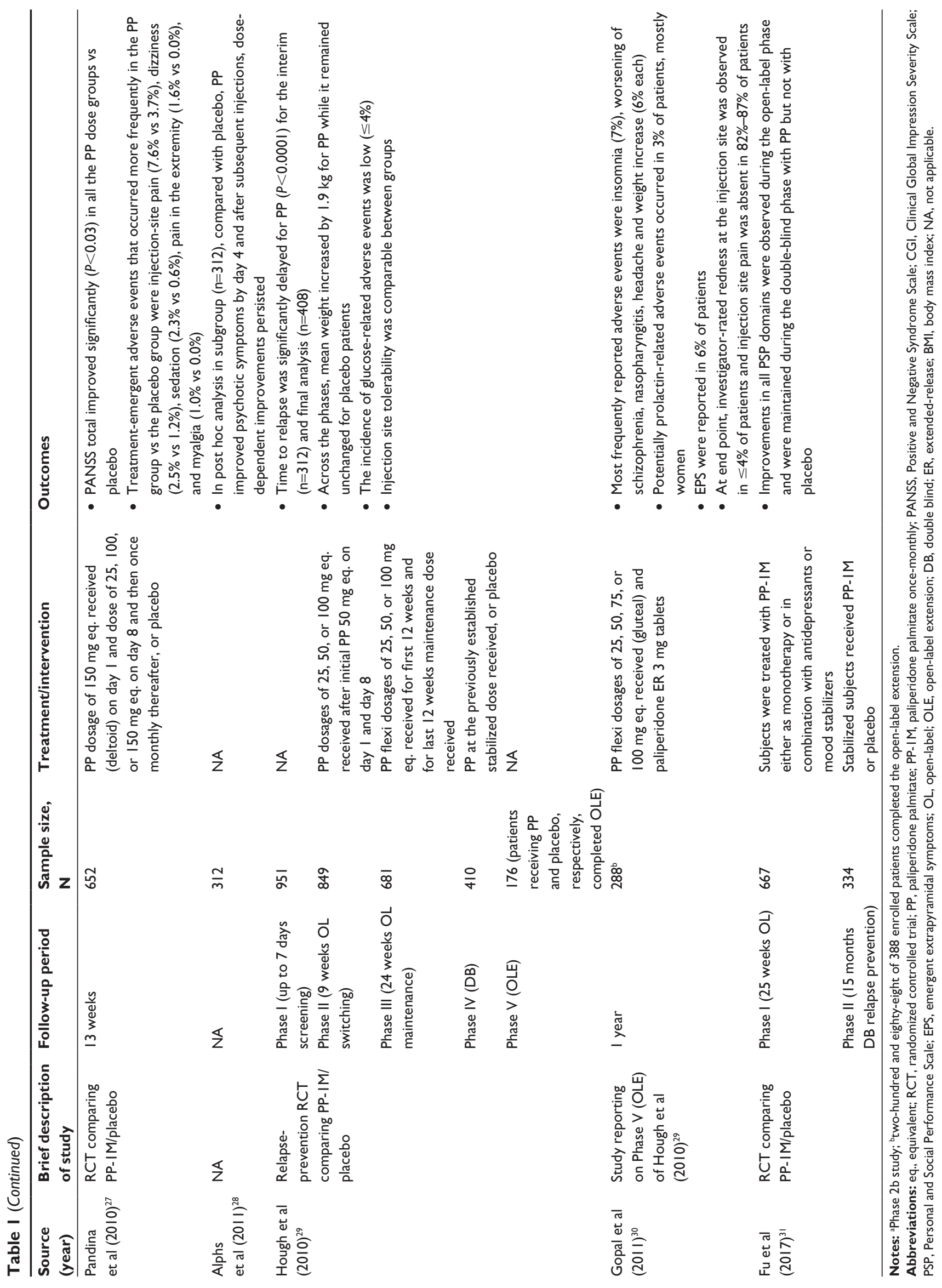


events. PP-treated patients experienced more parkinsonism than placebo. ${ }^{24}$ A 13-week RCT evaluated the efficacy, safety, and tolerability of fixed doses of 25,50 , and $100 \mathrm{mg}$ eq. PP-1M vs placebo was given as gluteal injections on days 1 and 8, then every 4 weeks (days 36 and 64) in patients with schizophrenia $(\mathrm{n}=518)$. All PP dose groups showed significant improvement vs placebo in the PANSS total score $(P<0.001)$ and Clinical Global Impression (CGI) Severity scores $(P \leq 0.006)$, but not in the Personal and Social Performance (PSP) scale score. The incidence of treatment-emergent adverse events was similar between groups, as were changes in parkinsonism and body mass index. ${ }^{25}$ Another 13-week RCT evaluated the efficacy and safety of PP-1M (50, 100, or $150 \mathrm{mg}$ eq.) vs placebo administered as monthly gluteal injections after two initial doses given 1 week apart in acutely symptomatic patients with schizophrenia $(n=388)$. As only 30 participants were randomized to the $150 \mathrm{mg}$ eq. dose group, definitive conclusions could not be drawn from the results of that group. PANSS total change scores were significantly better than placebo only for the $100 \mathrm{mg}$ eq. group $(P=0.02)$. Both the PP $50(P=0.004)$ and $100 \mathrm{mg}$ eq. $(P<0.001)$ groups showed significant improvement in the PSP score vs placebo. Adverse events reported more frequently in the PP groups than with placebo were headache, vomiting, extremity pain, and injection site pain. ${ }^{26}$ Another 13-week RCT assessed the efficacy and the safety of a dosing regimen for PP-1M that was revised from earlier studies for patients with acutely exacerbated schizophrenia $(n=652)$. Patients received an injection of PP $150 \mathrm{mg}$ eq. or placebo in the deltoid muscle on day 1 and the assigned fixed dose (PP 25, 100, or $150 \mathrm{mg}$ eq.) or placebo in the deltoid or gluteal on day 8 and then once monthly. Target plasma levels were achieved by day 8 in the PP groups. PANSS total scores improved significantly $(P<0.03)$ in all the PP dose groups vs placebo. Common treatment-emergent adverse events that occurred more frequently in the PP group vs the placebo group were injection site pain $(7.6 \%$ vs $3.7 \%)$, dizziness ( $2.5 \%$ vs $1.2 \%)$, sedation $(2.3 \%$ vs $0.6 \%)$, pain in the extremity $(1.6 \%$ vs $0.0 \%)$, and myalgia (1.0\% vs $0.0 \%) .{ }^{27} \mathrm{~A}$ post hoc analysis in a subgroup $(n=312)$ of markedly to severely ill patients in the abovementioned study found that compared with placebo, PP improved psychotic symptoms by day 4 , and after subsequent injections, dose-dependent improvements persisted. ${ }^{28}$

\section{Randomized, controlled trial vs placebo for maintenance treatment}

Efficacy of PP-1M vs placebo in relapse prevention was assessed in a complex study design. Patients with a PANSS total score $<120$ were transitioned from previous antipsychotics to PP during a 9-week, open-label phase. Two intramuscular injections of $50 \mathrm{mg}$ eq. PP were given 1 week apart, followed by flexibly dosed monthly injections $(25,50$, or $100 \mathrm{mg}$ eq.). Stable patients (PANSS total score $\leq 75$ ) then entered a 24-week maintenance phase, following which they were randomized to either continue PP at the stabilized dose or begin placebo in the double-blind phase. Time-torelapse, the primary end point, was significantly delayed for PP $(P<0.0001)$ for the interim $(n=312)$ and final analysis $(\mathrm{n}=408)$. Across the phases, mean weight increased by $1.9 \mathrm{~kg}$ for PP while it remained unchanged for placebo patients. The incidence of glucose-related adverse events was low $(\leq 4 \%)$. Injection site tolerability was comparable between groups. ${ }^{29}$ In a 1-year open-label extension to this study, the safety and tolerability of PP were assessed. Patients received gluteal injections of PP-1M starting at $50 \mathrm{mg}$ eq., followed by flexible dosing of 25, 50, 75, or $100 \mathrm{mg}$ eq. Two-hundred and eighty eight of the 388 enrolled patients completed the open-label extension. The most frequently reported adverse events were insomnia (7\%), worsening of schizophrenia, nasopharyngitis, headache, and weight increase ( $6 \%$ each). Potentially, prolactin-related adverse events occurred in 3\% of patients, who were mostly women. Emergent extrapyramidal symptoms (EPS) were reported in $6 \%$ of patients. At the end point, investigator-rated redness at the injection site was observed in $\leq 4 \%$ of patients and injection site pain was rated by investigators as absent in $82 \%-87 \%$ of patients. ${ }^{30}$

A RCT evaluated the effect of PP-1M on the PSP scale in patients with schizoaffective disorder experiencing an acute exacerbation of psychotic and mood symptoms. Subjects were treated with PP-1M either as monotherapy or in combination with antidepressants or mood stabilizers during a 25-week open-label phase $(n=667)$, and stabilized subjects $(n=334)$ were randomly assigned to PP-1M or placebo in a 15-month double-blind relapse-prevention phase. Improvements in all PSP domains were observed during the openlabel phase and were maintained during the double-blind phase with PP but not with placebo. ${ }^{31}$

\section{Comparative randomized, controlled trials PP-IM vs risperidone long-acting injectable}

A 53-week, Phase III RCT was designed to assess the noninferiority of PP-1M to risperidone long-acting injectable (RLAI) in 747 patients with schizophrenia. Acutely symptomatic patients $(n=749)$ were randomized to gluteal 
injections of either PP $50 \mathrm{mg}$ eq. on days 1 and 8, followed by flexible dosing (25-100 mg eq.) once-monthly, or RLAI 2-weekly, $25 \mathrm{mg}$ on days 8 and 22 followed by flexible dosing (25-50 mg) starting from day 36, with oral risperidone supplementation. Mean (SD) change from baseline to end point in PANSS total score was -11.6 (21.22) for PP and -14.4 (19.76) for RLAI, and least-squares means difference was -2.6 (95\% CI: $-5.84,0.61)$. PP's failure to demonstrate comparable efficacy to RLAI was attributed to the suboptimal initiation dosing regimen which resulted in lower median plasma levels of the active moiety in PP-treated patients. Tolerability profiles of both treatments were comparable to previous studies. ${ }^{32}$

Another RCT, also designed to assess noninferiority of PP vs RLAI, was conducted in 1,220 patients with schizophrenia over 13 weeks. The dosing regimen for the PP arm was as follows: deltoid injections on day 1 (150 mg eq.), day 8 (100 mg eq.), and flexible dosing as deltoid or gluteal injections on day 36 (50 mg eq. or $100 \mathrm{mg}$ eq.) and day 64 (50 mg eq., $100 \mathrm{mg}$ eq., or $150 \mathrm{mg}$ eq.). For the RLAI arm, patients received gluteal injections on days 8 and $22(25 \mathrm{mg})$, days 36,50 (25 or $37.5 \mathrm{mg}$ ), and days $64,78(25,37.5$, or $50 \mathrm{mg}$ ), and oral supplementation with risperidone on days 1 to 28 . Mean (SD) PANSS total score change from baseline to end point decreased similarly in both groups: PP (-18.6 [15.45]) and RLAI (-17.9 [14.24]). PP treatment was noninferior to RLAI (point estimate [95\% CI]: 0.4 [-1.62, 2.38]). The tolerability and safety of PP was generally similar to that of RLAI. ${ }^{33}$ This dosing regimen subsequently became the recommended initiation dosing procedure for PP-1M. In a post hoc subgroup analysis in markedly to severely ill subjects $(n=292)$ from this study, PANSS total scores improved significantly in both arms, starting from day 4 through to end point, ${ }^{34}$ and in another post hoc, subgroup analysis from this study, no significant differences in the mean weight change, most metabolic parameters, or mean efficacy measures were observed over the 13 weeks of treatment. ${ }^{35}$

An open-label, rater-blinded, RCT evaluated noninferiority of PP-1M to 2-weekly RLAI in 452 Chinese patients with acute schizophrenia. PP-treated patients received deltoid injections on day 1 (150 mg eq.) and day 8 (100 mg eq.), and then once-monthly deltoid or gluteal injections, flexibly dosed (50, 100, or $150 \mathrm{mg}$ eq.). RLAI-treated patients received 2-weekly gluteal injections, flexibly dosed (25, 37.5 , or $50 \mathrm{mg}$ ), and oral risperidone supplementation at initiation and with RLAI dose increases. Mean (SD) change from baseline to end point in PANSS total scores was -23.6 (16.28) for the PP group and -26.9 (15.43) for RLAI group. $\mathrm{PP}$ was noninferior to RLAI (least-squares mean difference
[95\% CI]: $-2.3[-5.20,0.63])$. The incidence of treatmentemergent adverse events was similar between the two groups. The most common adverse events were akathisia, tremor, and insomnia. ${ }^{36}$

A 6-month, open-label RCT compared the effects of RLAI and PP-1M on social functioning in a small sample of patients with schizophrenia $(n=30)$. The PP-treated patients showed a greater degree of total social functioning, independent life competence, and performance as compared to the RLAI group. ${ }^{37}$ In the same sample, effects of PP-1M and RLAI on cognitive functions were compared. The results suggested that PP might improve attention and processing speed more than RLAI. ${ }^{38}$ A pragmatic, randomized, openlabel, flexible dose study compared PP-1M (n=226) with oral antipsychotics $(n=218)$ over 15 months in patients with schizophrenia and a history of contact with the criminal justice system. The mean cumulative function of treatment failure events and institutionalizations differed significantly in favor of PP compared with oral antipsychotics $(P=0.007$ and $P=0.005$, respectively). ${ }^{39}$ To assess patient satisfaction with switching from oral antipsychotics to a long-acting injectable antipsychotic formulation, a 21-week, open-label RCT was conducted in 154 patients with schizophrenia dissatisfied with current oral atypical antipsychotics. Participants were randomized to either immediate or delayed switching to PP-1M. Medication satisfaction improved significantly in both groups, with comparable efficacy and tolerability (Table 2$){ }^{40}$

\section{PP-IM vs aripiprazole once-monthly $400 \mathrm{mg}$}

A randomized, noninferior, open-label, rater-blinded, head-to-head comparison of PP-1M and aripiprazole oncemonthly $400 \mathrm{mg}$ (AOM 400) was conducted over 28 weeks in 295 patients with schizophrenia, with the HeinrichsCarpenter Quality-of-Life Scale (QLS) score as the primary outcome. Sixty-eight percent of AOM 400 and $57 \%$ of PP patients completed 28 weeks of treatment. AOM-treated patients showed significantly greater improvements in the QLS total score (4.67 [95\% CI: 0.32, 9.02], $P=0.04$ ). There were also significantly greater improvements in CGI Severity scale and the Investigator's Assessment Questionnaire for AOM 400 vs PP. Common treatment-emergent adverse events in the treatment continuation phase were more frequent with PP vs AOM $400 .{ }^{41}$

\section{PP-IM vs haloperidol decanoate}

A double-blind RCT was conducted in 311 patients with schizophrenia or schizoaffective disorder who were clinically 


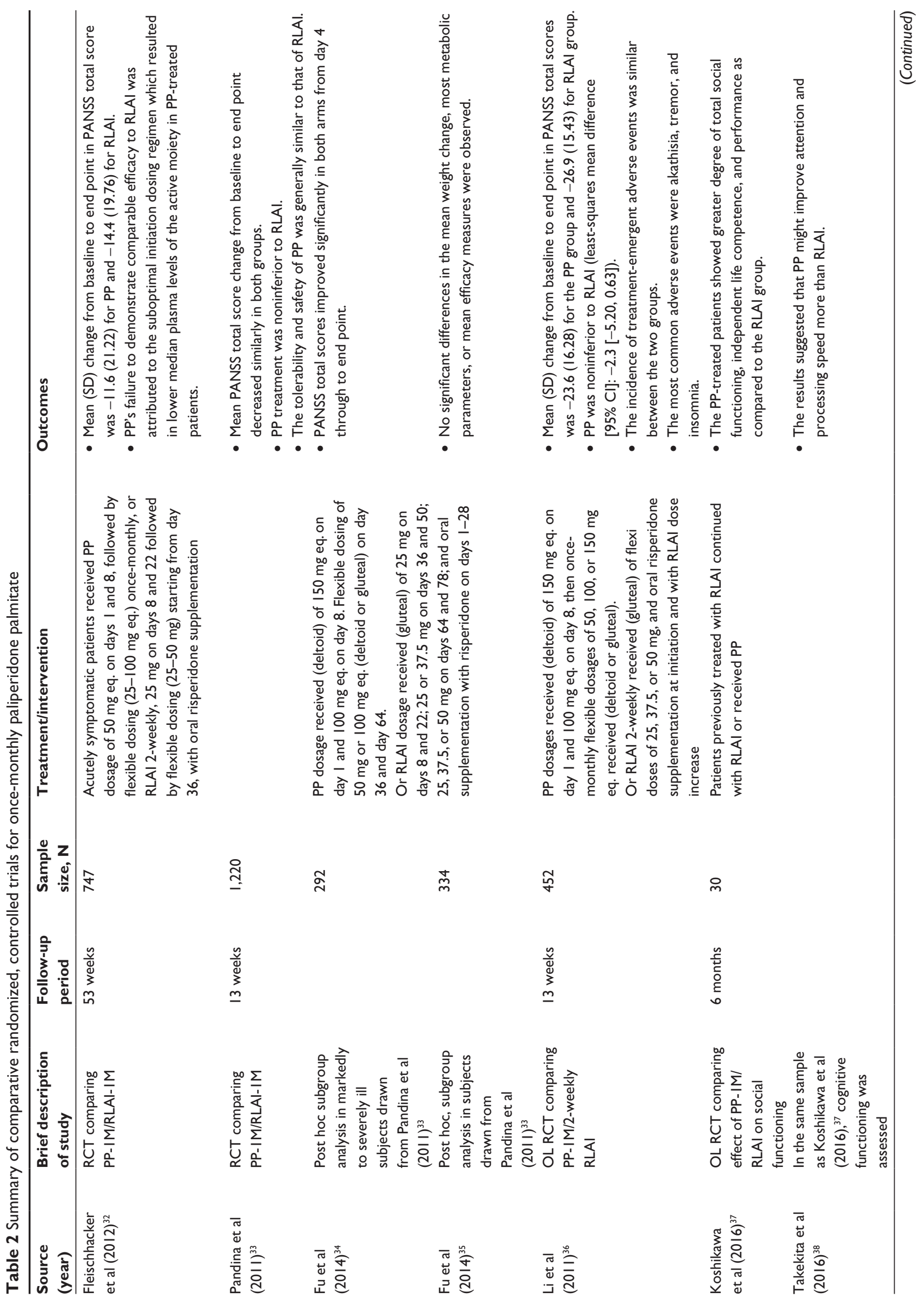




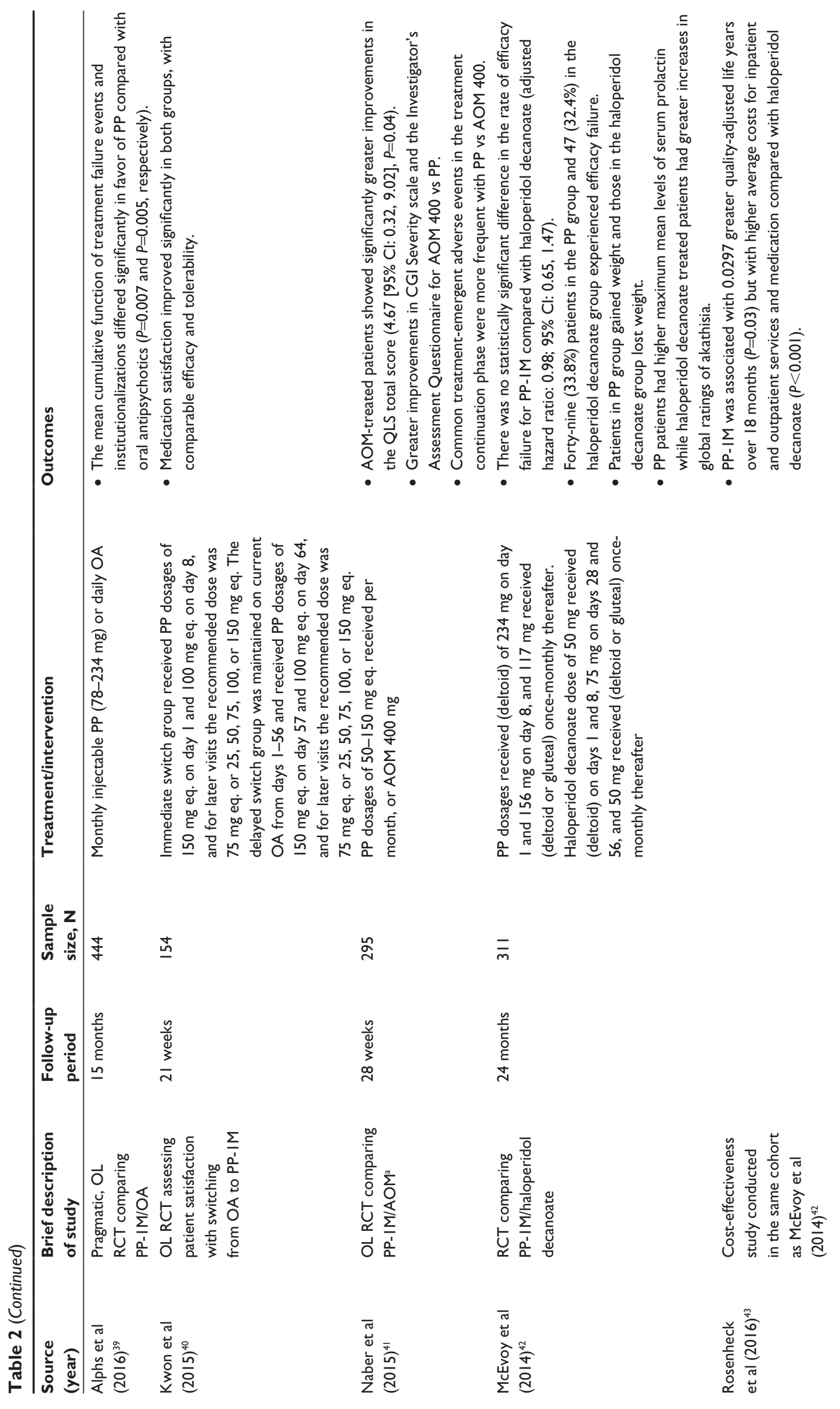



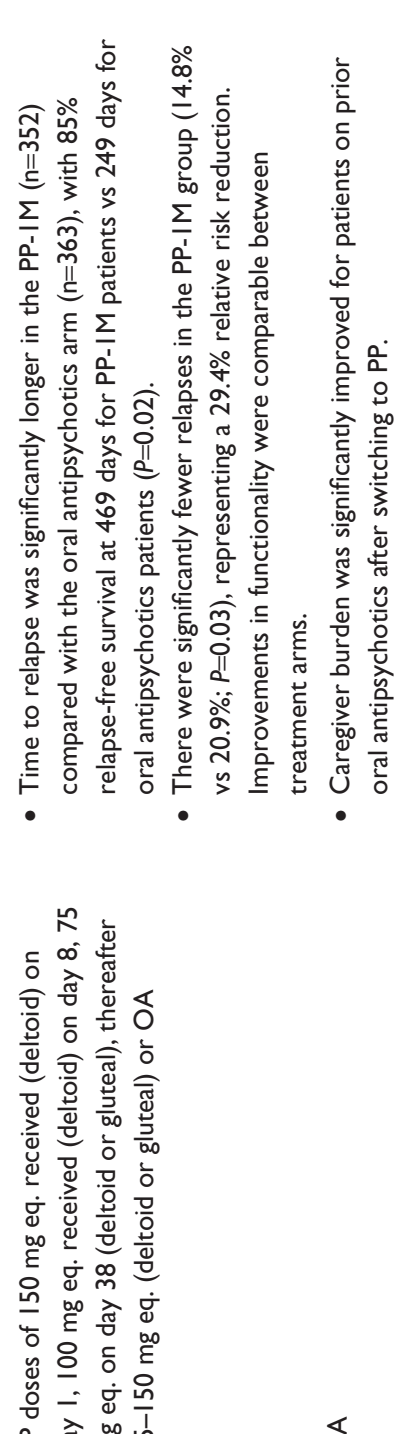

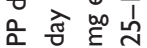

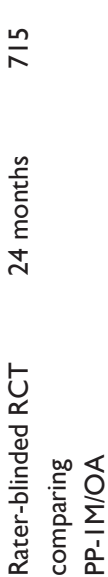

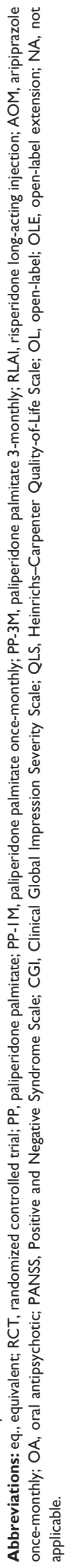

assessed to be at risk of relapse, for up to 24 months, to compare the effectiveness of PP-1M vs haloperidol decanoate. The primary outcome measure was efficacy failure, defined as a psychiatric hospitalization, a need for crisis stabilization, increase in frequency of outpatient visits, a clinician's decision that oral antipsychotic could not be discontinued within 8 weeks after starting the LAIs, or a clinician's decision to discontinue due to inadequate therapeutic benefit. There was no statistically significant difference in the rate of efficacy failure for PP-1M compared with haloperidol decanoate (adjusted hazard ratio: 0.98; 95\% CI: 0.65, 1.47). Forty-nine (33.8\%) patients in the PP group and 47 (32.4\%) in the haloperidol decanoate group experienced efficacy failure. Overall, participants in the PP group gained weight and those in the haloperidol decanoate group lost weight. The PP-treated patients had significantly higher maximum mean levels of serum prolactin while haloperidol decanoatetreated patients had significantly greater increases in global ratings of akathisia. ${ }^{42}$ In a relative cost-effectiveness study in the same cohort, mixed-model analysis showed that PP-1M was associated with 0.0297 greater quality-adjusted life years over 18 months $(P=0.03)$, but with higher average costs for inpatient and outpatient services and medication compared with haloperidol decanoate $(P<0.001){ }^{43}$

\section{PP-IM vs oral antipsychotics in recent- onset schizophrenia}

An RCT assessed the efficacy of PP-1M vs physician's choice of oral antipsychotics for relapse prevention in patients with a recent diagnosis of schizophrenia (within 5 years). Patients who met predefined response criteria were entered into the 24-month rater-blinded treatment phase. Time to relapse was significantly longer in the PP-1M ( $\mathrm{n}=352)$ compared with the oral antipsychotics arm $(n=363)$, with $85 \%$ relapse-free survival at 469 days for PP-1M patients vs 249 days for oral antipsychotics patients $(P=0.02)$. There were significantly fewer relapses in the PP-1M group (14.8\% vs $20.9 \%$; $P=0.03$ ), representing a $29.4 \%$ relative risk reduction. Improvements in functionality were comparable between treatment arms. ${ }^{44}$ In a pooled analysis of two RCTs (PP-1M and PP-3M), predictors of caregiver burden were evaluated ( $\mathrm{n}=1,498$ caregivers). Caregiver burden was significantly improved for patients on prior oral antipsychotics after switching to PP. ${ }^{45}$

\section{Observational studies and uncontrolled pragmatic trials with paliperidone once-monthly}

In a naturalistic study conducted over 1 year to determine the factors predicting continuation with PP treatment $(n=200)$, 
initiation as an outpatient $(P=0.001)$, being switched from risperidone $(P=0.03)$, and correct initiation dosing $(P=0.02)$ were significantly associated with a lower likelihood of discontinuation. ${ }^{46}$ Another naturalistic study explored efficacy, safety, and tolerability of PP-1M initiated shortly after hospital admission in 367 patients hospitalized for an exacerbation of schizophrenia, over 6 weeks. Significant improvements were observed in psychotic symptoms $(P<0.0001)$ and PSP scores $(P<0.0001)$, and the treatment was well tolerated. ${ }^{47}$

A study comparing real-world health care costs and resource utilization between patients with schizophrenia treated with PP-1M and oral atypical antipsychotics found that patients treated with PP had lower inpatient costs and admission rates, although total health care costs were not significantly different. ${ }^{48}$

A retrospective mirror-image observational study assessed the effects of PP-1M on acute inpatient hospitalization rates in 66 patients with schizophrenia who had received PP-1M for at least 1 year. The mean number of acute admissions fell from 0.86 in the year before PP initiation to 0.23 in the following year $(P=0.001) .{ }^{49}$

An observational, claims-based study examined the frequency and duration of concurrent oral prescriptions in 340 patients receiving LAI formulations of antipsychotics (first and second generation) with a recent history of nonadherence and hospitalization for schizophrenia. The lowest rate of concurrent oral prescribing (58.8\%) was found with PP, whereas the highest rate was with RLAI $(88.9 \%) .{ }^{50}$

A single-arm, multicenter, open-label, 6-month study in patients with schizophrenia assessed the tolerability, safety, and efficacy of flexible doses of PP-1M in a subset of nonacute but symptomatic patients previously unsuccessfully treated with oral antipsychotic agents $(n=593)$. PANSS total scores decreased from 71.5 (14.6) at baseline to 59.7 (18.1) at end point $(P<0.0001)$. PSP scores improved significantly from baseline to end point $(P<0.0001) .{ }^{51}$ In the same study, but assessing only patients who switched to PP-1M after unsuccessful treatment with RLAI or conventional depot antipsychotics $(n=231)$, significant reductions in PANSS total scores were observed after switching $(P=0.01) .{ }^{52}$ A third subset analysis of the same study was conducted in patients with acute symptoms of schizophrenia following switching from previously unsuccessful treatment with oral antipsychotics who were switched to PP-1M (n=212). Significant improvements in PANSS total score were observed from day 8 onwards $(P<0.0001)$. PP was also associated with significant improvements in secondary measures of symptom severity, subjective well-being, medication satisfaction, illness-related disorders of activity and participation, and patient functioning $(P<0.0001)$. PP was generally well tolerated, with significant reductions in Extrapyramidal Symptom Rating Scale total score $(P<0.0001) .{ }^{53}$ A 25 -week, open-label study evaluated safety and efficacy of PP-1M in 353 Chinese patients with schizophrenia. PANSS total scores improved significantly $(P<0.0001)$, as did PSP scores $(P<0.0001)$. Most frequently reported emergent adverse events were extrapyramidal disorder $(15.3 \%)$, akathisia $(10.5 \%)$, blood prolactin increase (8.8\%), and insomnia (5.4\%). Eight deaths were reported, including four completed suicides. ${ }^{54}$

A retrospective cohort study compared data on rehospitalization patterns and associated institutional costs after inpatient treatment with PP-1M or oral antipsychotic therapy. In the first 12 months after hospital discharge, the risk of rehospitalization and emergency room use was significantly lower in the PP-1M cohort than in the oral antipsychotic cohort $(P<0.0001)$, and institutional costs during the first 6 months after discharge were significantly lower in the PP-1M cohort $(P<0.0001){ }^{55}$

A prospective observational study over 3 months assessed the effect of switching from RLAI to PP-1M on sexual function and prolactin levels in a small sample $(n=11)$ of patients with psychosis who developed hyperprolactinemia on RLAI and who were then switched to PP. There was a significant decrease in serum prolactin levels $(P=0.04)$ and a fourfold reduction in clinically significant sexual dysfunction. ${ }^{56}$

A retrospective, observational cohort study using patient claims data compared effectiveness evidence for PP-1M vs oral atypical antipsychotics. Emergency room visits and hospitalization rates were lower in PP-1M patients, although hospitalizations did not achieve statistical significance. ${ }^{57}$

In an observational study, 200 consecutive patients prescribed PP in normal practice were followed up. After 1 year, $65 \%$ of patients were still receiving PP. The number of admissions to hospital in the year following PP initiation was 0.49 /patient compared with $0.69 /$ patient/year for the 3 years before initiation $(P=0.0001)$. The median number of bed days for the 3 years before PP initiation was 21.50/year and in the year following PP initiation, it was $0 .^{58}$

In a noninterventional, observational study of consecutive patients treated with PP-1M ( $=300), 38.7 \%$ completed 2 years of continuous treatment and a further $7.6 \%$ patients discontinued PP but restarted after $>2$ months. Prior treatment with risperidone reduced the risk of discontinuation by $39 \%(P=0.004)$ and having treatment initiated as an outpatient by $49 \%(P=0.001)$. The risk of discontinuation was 
increased by $63 \%$ when the reason for prescribing PP was poor tolerability of the prior treatment $(P=0.028) .{ }^{59}$

A part-prospective mirror-image study examined outcomes 2 years before starting PP-1M and 2 years after $(n=225)$. At study end point, $41.8 \%$ of patients were still receiving PP-1M. The number of admissions fell from 1.80 in the 2 years before starting PP- $1 \mathrm{M}$ to 0.81 in 2 years following initiation or discharge $(P<0.001)$. Total bed days were reduced from 79.6 in the 2 years before to 46.2 in the 2 years after initiation or discharge $(P<0.001){ }^{60}$

The relapse risk following a switch from RLAI to PP-1M $(n=188)$ vs a switch from RLAI to oral antipsychotics $(n=131)$ was examined using information from a Medicaid database. Patients who switched from RLAI to PP had fewer events (hospitalization or emergency room visit) (26 vs 32), longer time to an event (mean 70 vs 47 days), and lower risk of relapse $(P=0.02)$ compared with those who switched from RLAI to oral antipsychotics. ${ }^{61}$

A naturalistic cohort of all identifiable patients who initiated PP-1M in a specific region in the United Kingdom $(n=179)$ was assessed. Sixty percent of patients continued PP-1M beyond 12 months. Schizophrenia diagnosis, fewer inpatient days after initiation, dose adjustment up or down, and a higher maintenance dose were associated with treatment continuation. ${ }^{62}$

In an 18-month, open-label, noncomparative study from the Asia-Pacific region, patients with recent-onset schizophrenia ( $\leq 5$ years) who were unsatisfactorily treated with previous oral antipsychotics $(n=521)$ were treated with flexible doses of PP-1M. PANSS total scores improved significantly $(P<0.0001)$. There were greater improvements among patients with more severe symptoms. In a mirror analyses subset $(n=474)$, PP significantly reduced mean number of hospitalization days/person/year as well as percentage of patients requiring hospitalization in past 12 months $(P<0.0001)$. Adverse events $(\geq 15 \%)$ were EPS $(31.3 \%)$, injection site pain (18.6\%), and insomnia (15.2\%). ${ }^{63}$

A retrospective claims-based analysis of Medicaid patients with schizophrenia compared all-cause health care utilization and costs between patients treated with PP-1M ( $\mathrm{n}=722)$ vs atypical oral antipsychotics $(n=722)$. Over 12 months, PP-1M patients were less likely to discontinue treatment $(30.6 \%$ vs $39.5 \%, P<0.001)$ or switch to a new therapy (21.6\% vs $27.7 \%, P=0.007)$ and had fewer inpatient visits (5.0 vs 7.9, $P<0.001$ ), lower mean hospitalization days (15.0 vs 27.7 days, $P<0.001$ ), and inpatient costs (US $\$ 5,060$ vs US $\$ 10,880, P=0.001)$. Pharmacy costs were significantly higher in the PP-1M cohort $(P<0.001)$, although total costs were not significantly different $(P=0.853) \cdot{ }^{64}$ Another study in patients recently diagnosed with schizophrenia compared adherence, health care resource utilization, and Medicaid spending between schizophrenia patients treated with PP-1M $(n=2,053)$ vs those treated with oral atypical antipsychotics $(\mathrm{n}=22,247)$. Patients treated with PP-1M had better adherence $(P<0.001)$ and required less use of concomitant psychiatric medications. Lower medical costs associated with PP-1M outweighed the higher pharmacy costs, with similar total health care costs across the groups. ${ }^{65}$

An international, multicenter, retrospective chart review of medical records of adult patients who were newly diagnosed ( $<1$ year) with schizophrenia and who had received continuous treatment with PP-1M for $\geq 12$ months in naturalistic clinical settings $(\mathrm{n}=84)$ was undertaken. Of the patients, $79.2 \%$ had $\geq 20 \%$ improvement and $47.2 \%$ had a $\geq 50 \%$ improvement in PANSS total scores. PSP scores improved significantly in $53.3 \%$. Most adverse drug reactions were mild or moderate in severity (Table 3 ). ${ }^{66}$

\section{Three-monthly paliperidone palmitate}

In 2015, a new formulation, 3-monthly injection of prolonged release suspension of PP (PP-3M), was approved for the maintenance treatment of schizophrenia in adult patients by the European Medicines Agency (Trevicta ${ }^{\circledR}$, Janssen Pharmaceutica NV), following earlier approval in the United States by the Food and Drug Administration (Invega Trinza ${ }^{\circledR}$, Janssen Pharmaceutica NV). Pharmacokinetic studies support the feasibility of administering PP on a 3-monthly basis, subsequent to stabilization of treatment with the administration of four once-monthly injections of PP-1M, at doses 3.5 times higher than the last dose of PP-1M. The dose range for PP-3M is $175,300,450$, and $525 \mathrm{mg}$ eq. ${ }^{67}$

A relapse-prevention RCT compared PP-3M to placebo in 506 patients with schizophrenia. The trial comprised four phases, namely a 3-week screening phase; a 17-week, flexibledose, open-label transition phase; a 12-week open-label maintenance phase; and an open-ended double-blind phase. Patients received once-monthly doses of PP-1M $(50,75,100$, or $150 \mathrm{mg}$ eq.) during the transition phase, followed by a single dose of PP-3M (at 3.5 times the stabilized dose of PP-1M) during the maintenance phase. Stable patients were then randomized to receive either a fixed dose of PP-3M $(175,263,350$, or $525 \mathrm{mg}$ eq.) or placebo once every 3 months during the double-blind phase. In the preplanned interim analysis, time to relapse was significantly longer in the PP-3M group vs the placebo group $(P=0.001)$. PANSS total score and factor analysis derived 


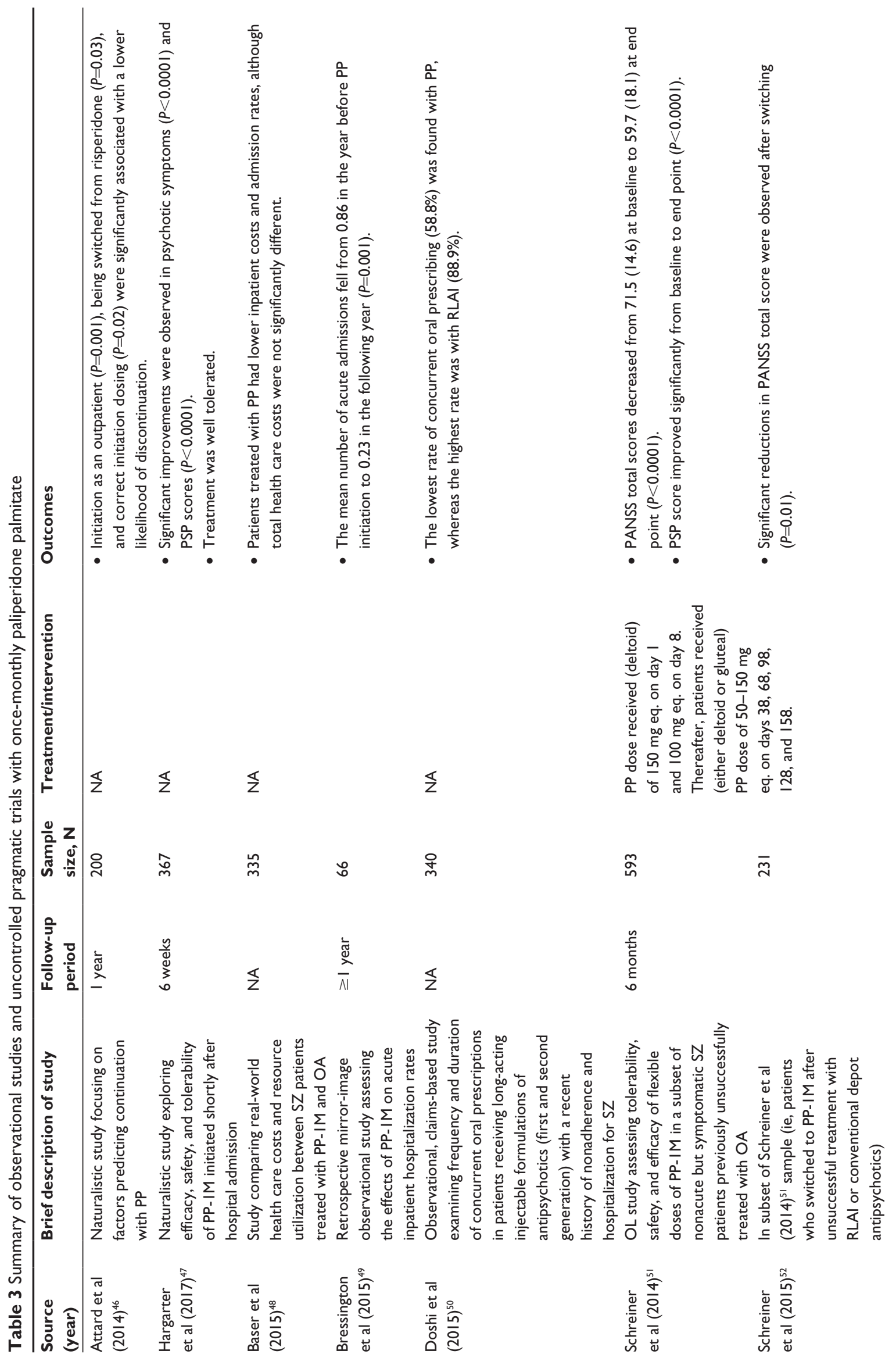



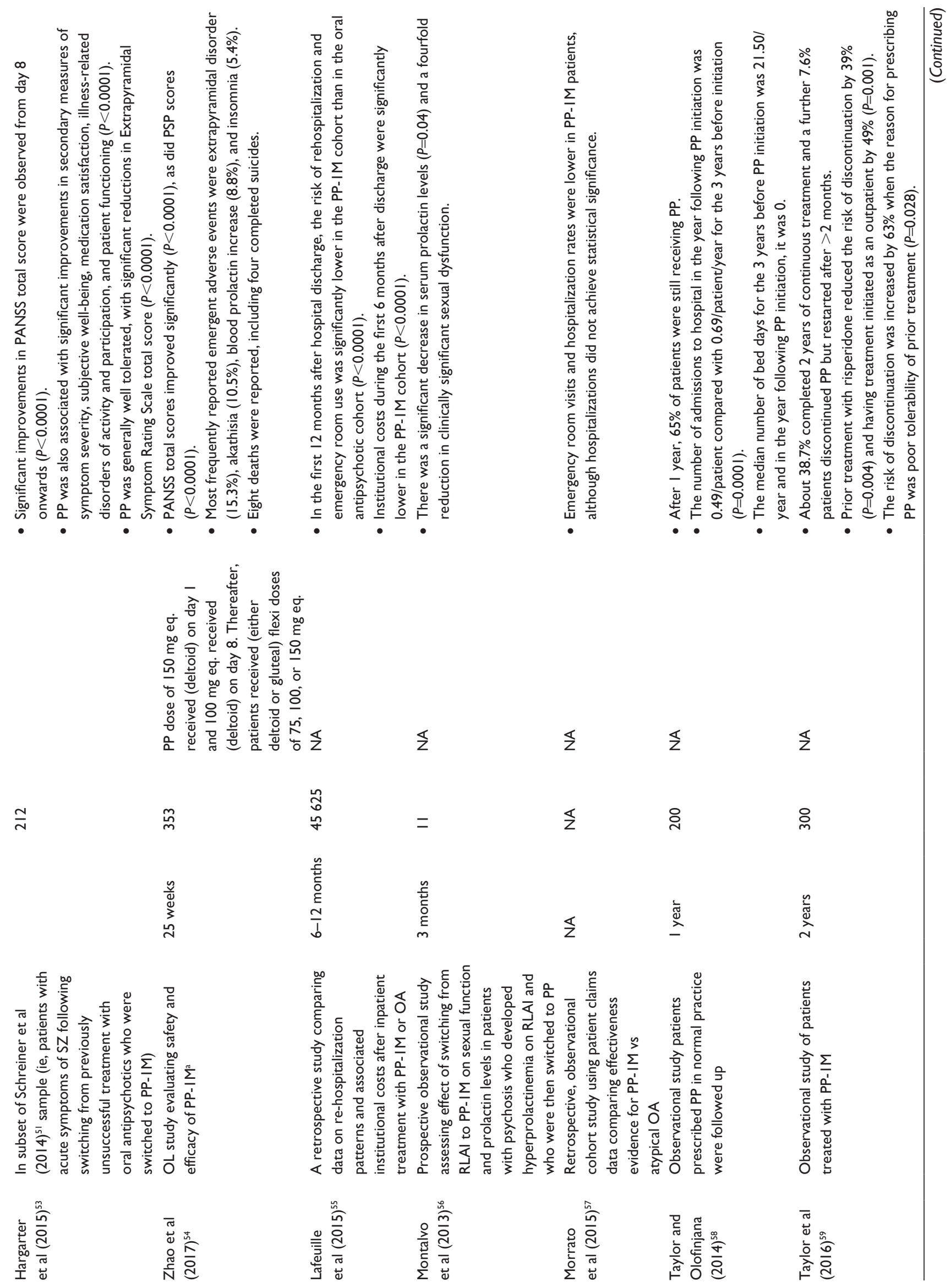


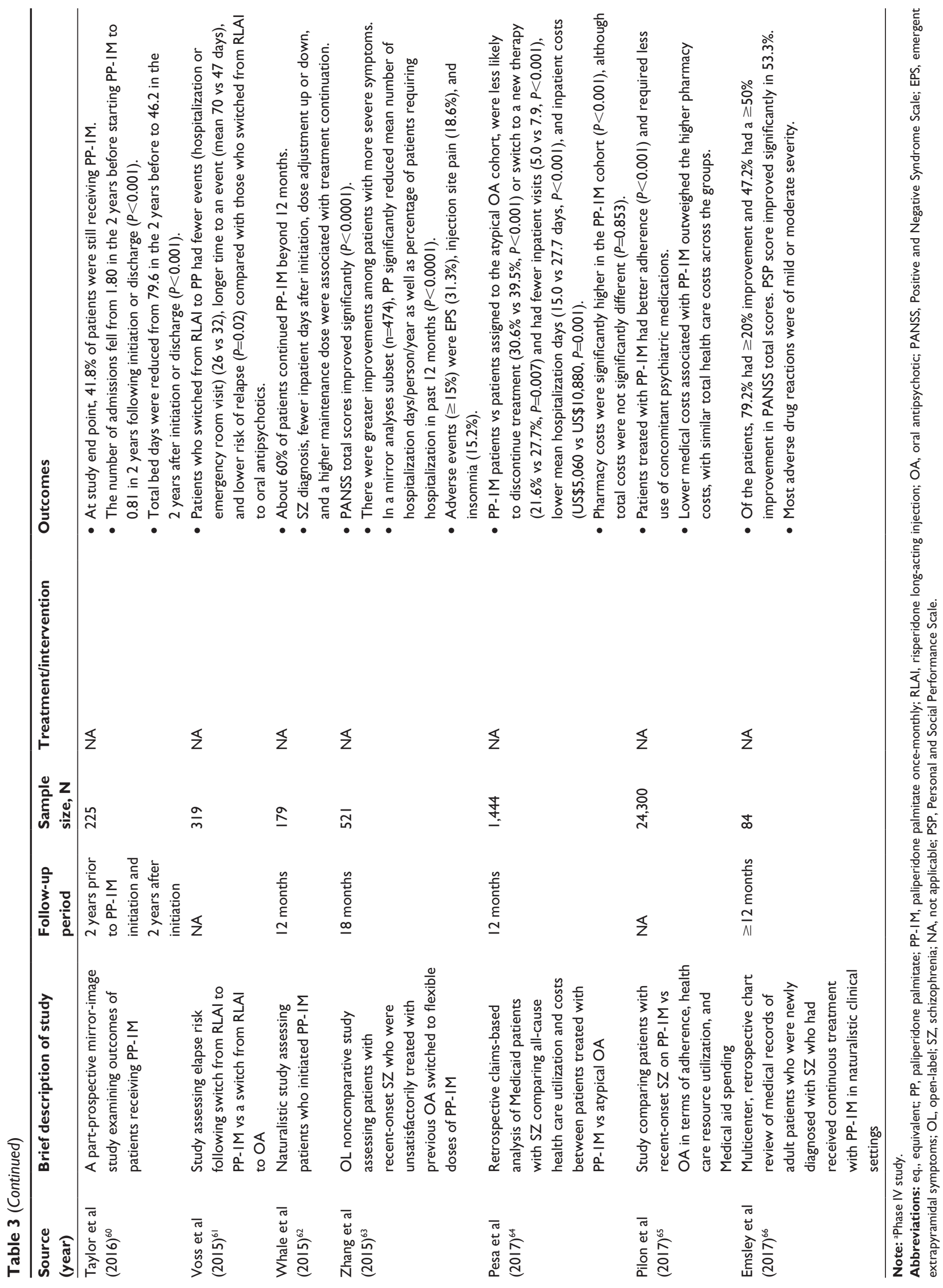


domain score reductions were greater in PP-3M than placebo $(P=0.01)$. In the double-blind phase, the most frequently reported adverse events in the PP-3M vs placebo groups were headache ( $9 \%$ vs $4 \%$ ), weight gain ( $9 \%$ vs 3\%), nasopharyngitis ( $6 \%$ vs $1 \%$ ), and akathisia (4\% vs $1 \%){ }^{68}$

A double-blind RCT was designed to test the noninferiority of PP-3M formulation to PP-1M in 1,016 patients with schizophrenia who were previously stabilized on PP-1M. After screening and a 17-week, flexible-dosed, open-label phase, clinically stable patients were randomized to PP-3M (fixed-dose, 175, 263, 350, or $525 \mathrm{mg}$ eq. deltoid/gluteal) or PP-1M (fixed-dose, 50, 75, 100, or $150 \mathrm{mg}$ eq. deltoid/gluteal) for a 48-week double-blind phase. PP-3M was noninferior to PP-1M insofar as relapse rates were similar in both groups ( $8 \%$ vs $9 \%$ ), as were PANSS total score changes and PSP score changes. There were no clinically relevant differences in pharmacokinetic exposure between PP-3M and PP-1M. Both groups had similar tolerability profiles with weight gain as the most common treatment-emergent adverse event. ${ }^{69}$ A post hoc analysis assessed the rates of symptomatic and functional remission achieved following PP-3M vs PP-1M treatment in the same sample. In the PP-3M group, 50.3\% achieved symptomatic remission vs $50.8 \%$ in the PP-1M group. Functional remission was achieved in $42.5 \%$ in the PP-3M group and $43.9 \%$ in the PP-1M group..$^{70}$ Another post hoc analysis of a subset of patients from the same study assessed 510 East Asian participants. Again, the percentage of patients who relapsed was similar for PP-3M (10.2\%) and PP-1M (11.8\%), as was symptom reduction. ${ }^{71}$ Results from the abovementioned pivotal Phase III RCTs indicate that PP-3M was efficacious and generally well tolerated. The most frequently reported adverse events were anxiety, insomnia, weight gain, nasopharyngitis, and headache. While prolactin levels were increased, there was a low incidence of prolactin-related adverse events. ${ }^{72}$

In a post hoc study to evaluate the time to relapse following treatment discontinuation with oral paliperidone, PP-1M and PP-3M data were drawn from three similarly designed relapse prevention, placebo-controlled RCTs $(n=449)$. Median (95\% CI) days to relapse were 58 days (42-114 days) for oral paliperidone, 172 days (134-222 days) for PP-1M, and 395 days (274 days-not reached) for PP-3M $(P<0.0001){ }^{73}$ The delayed time to relapse for the LAI PP formulations, particularly PP-3M, may be important in clinical practice where treatment gaps frequently occur (Table 4).

\section{Discussion and conclusion}

Taken together, the abovementioned studies suggest that PP has good efficacy compared with placebo and is comparable

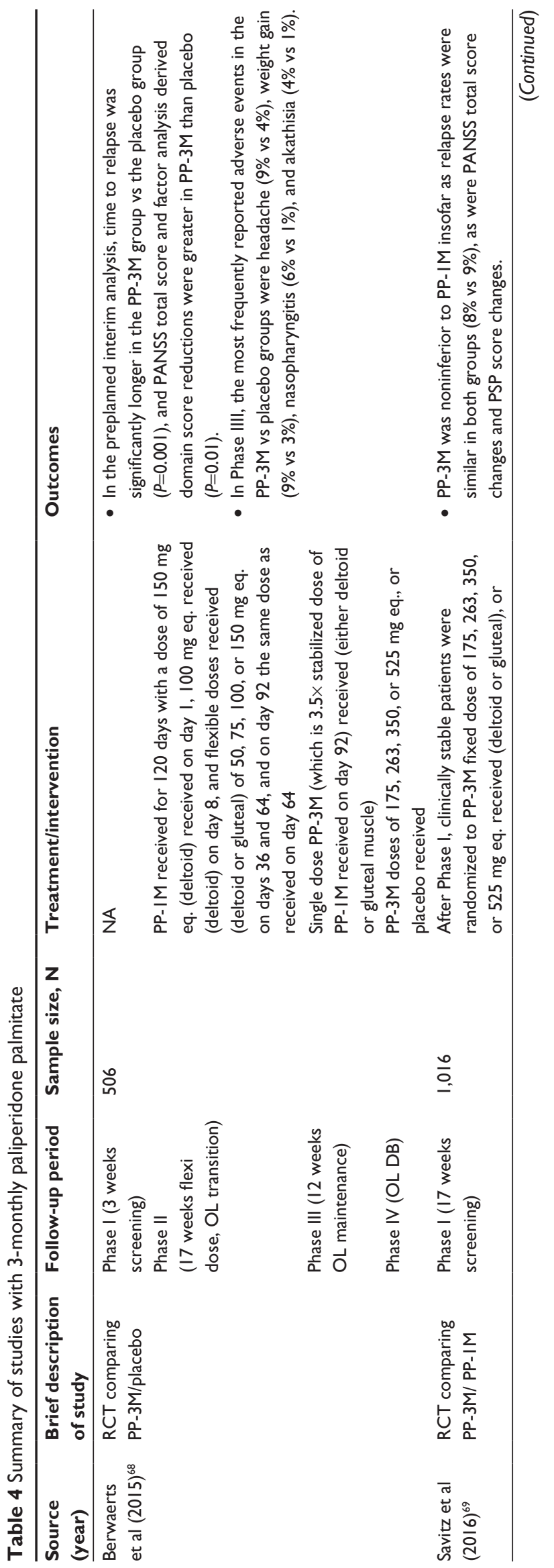




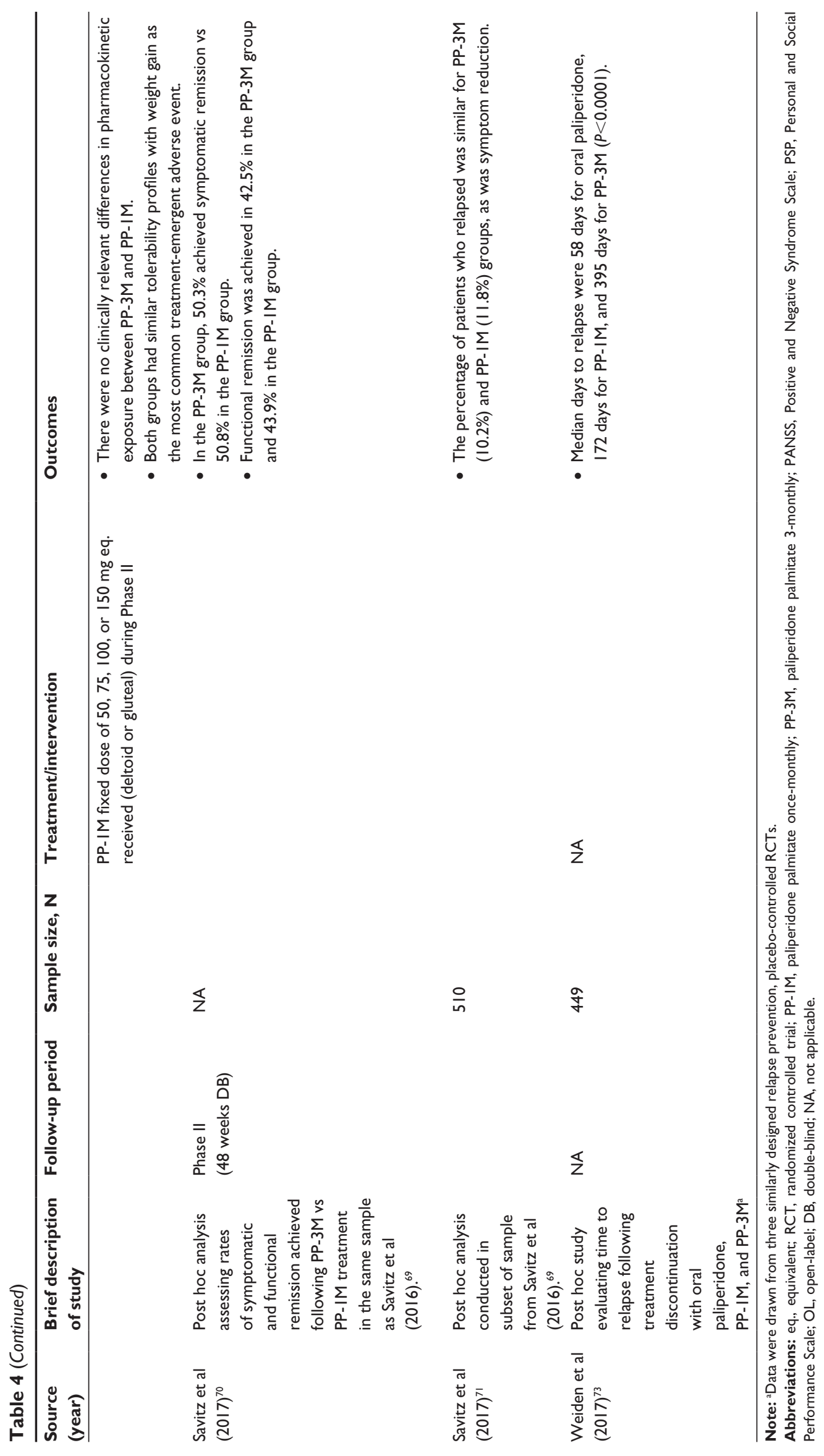


with risperidone. The tolerability profile of PP is similar to that of risperidone, with the most important side effects being prolactin elevation, weight gain, and extrapyramidal symptoms. Particularly in the early stages of illness, these side effects may be a significant barrier to effective treatment. Prolactin-related sexual dysfunction may be particularly relevant to younger people, and so too excessive weight gain.

Compared with RLAI, PP offers advantages of not requiring refrigeration or reconstitution prior to administration, or special needle kits. ${ }^{72}$ Additional advantages of PP include the following: First, there is a wealth of research data and clinical experience for both risperidone and paliperidone, so that the efficacy, safety, and tolerability profile of the compound is well known. Second, the availability of different formulations (PP-1M, PP-3M, and perhaps even longer-acting formulations in future) provides clinicians and patients with a variety of treatment options. A longer interval between injections may be important when aiming for recovery, where patient autonomy and independent living are key considerations. Third, injections may be given in either the deltoid or gluteal muscles, which may be of importance to some patients. Finally, the PP-1M dose-initiation procedure provides rapid onset of action, thereby circumventing the need for oral supplementation and enabling its use in acute settings. The importance of adhering to the recommended initiation dosing regimen needs to be emphasized. Earlier studies suggested that using a lower initial dose may compromise the early treatment response. ${ }^{32}$ Subsequent carefully conducted clinical trials and pharmacokinetic data confirmed the importance of the initial loading doses. In conclusion, PP is an important treatment option available to patients with schizophrenia and related illness, although cost considerations limit its use in resource-constrained environments.

\section{Disclosure}

In the past 3 years, Robin Emsley has participated in speakers/ advisory boards and received honoraria from Janssen, Lundbeck, Servier, and Otsuka. He has also received research funding from Janssen and Lundbeck. Sanja Kilian reports no conflicts of interest in this work.

\section{References}

1. Chong HY, Teoh SL, Wu DB, Kotirum S, Chiou CF, Chaiyakunapruk N. Global economic burden of schizophrenia: a systematic review. Neuropsychiatr Dis Treat. 2016;12:357-373.

2. Andreasen NC, Carpenter WT Jr, Kane JM, Lasser RA, Marder SR, Weinberger DR. Remission in schizophrenia: proposed criteria and rationale for consensus. Am J Psychiatry. 2005;162(3):441-449.

3. Emsley R, Chiliza B, Asmal L. The evidence for illness progression after relapse in schizophrenia. Schizophr Res. 2013;148(1-3):117-121.
4. Wunderink L, Nieboer RM, Wiersma D, Sytema S, Nienhuis FJ. Recovery in remitted first-episode psychosis at 7 years of follow-up of an early dose reduction/discontinuation or maintenance treatment strategy: long-term follow-up of a 2-year randomized clinical trial. JAMA Psychiatry. 2013;70(9):913-920.

5. Leucht S, Tardy M, Komossa K, et al. Antipsychotic drugs versus placebo for relapse prevention in schizophrenia: a systematic review and meta-analysis. Lancet. 2012;379(9831):2063-2071.

6. Zipursky RB, Menezes NM, Streiner DL. Risk of symptom recurrence with medication discontinuation in first-episode psychosis: a systematic review. Schizophr Res. 2014;152(2-3):408-414.

7. Jiang Y, Ni W. Estimating the impact of adherence to and persistence with atypical antipsychotic therapy on health care costs and risk of hospitalization. Pharmacotherapy. 2015;35(9):813-822.

8. Emsley R, Chiliza B, Asmal L, Harvey BH. The nature of relapse in schizophrenia. BMC Psychiatry. 2013;13:50.

9. Owen MJ, O'Donovan MC. Schizophrenia and the neurodevelopmental continuum: evidence from genomics. World Psychiatry. 2017;16(3): $227-235$.

10. McGlashan TH. Is active psychosis neurotoxic? Schizophr Bull. 2006;32(4):609-613.

11. Morgan C, Lappin J, Heslin M, et al. Reappraising the long-term course and outcome of psychotic disorders: the AESOP-10 study. Psychol Med. 2014;44(13):2713-2726.

12. Lieberman J, Jody D, Geisler S, et al. Time course and biologic correlates of treatment response in first-episode schizophrenia. Arch Gen Psychiatry. 1993;50(5):369-376.

13. Emsley R, Rabinowitz J, Medori R. Remission in early psychosis: rates, predictors, and clinical and functional outcome correlates. Schizophr Res. 2007;89(1-3):129-139.

14. Robinson D, Woerner MG, Alvir JM, et al. Predictors of relapse following response from a first episode of schizophrenia or schizoaffective disorder. Arch Gen Psychiatry. 1999;56(3):241-247.

15. Swartz MS, Stroup TS, McEvoy JP, et al. What CATIE found: results from the schizophrenia trial. Psychiatr Serv. 2008;59(5):500-506.

16. Pousa E, Ochoa S, Cobo J, et al. A deeper view of insight in schizophrenia: insight dimensions, unawareness and misattribution of particular symptoms and its relation with psychopathological factors. Schizophr Res. 2017;189:61-68.

17. Keith SJ, Pani L, Nick B, et al. Practical application of pharmacotherapy with long-acting risperidone for patients with schizophrenia. Psychiatr Serv. 2004;55(9):997-1005.

18. Emsley R, Chiliza B, Asmal L, Mashile M, Fusar-Poli P. Long-acting injectable antipsychotics in early psychosis: a literature review. Early Interv Psychiatry. 2013;7(3):247-254.

19. Jiang $\mathrm{Y}, \mathrm{Ni}$ W. Health care utilization and treatment persistence associated with oral paliperidone and lurasidone in schizophrenia treatment. J Manag Care Spec Pharm. 2015;21(9):780-792.

20. Corena-McLeod M. Comparative pharmacology of risperidone and paliperidone. Drugs R D. 2015;15(2):163-174.

21. Invega Sustenna ${ }^{\circledR}$ [Product Monograph]. Markham, ON: Janssen-Ortho Inc; 2010.

22. de Leon J, Wynn G, Sandson NB. The pharmacokinetics of paliperidone versus risperidone. Psychosomatics. 2010;51(1):80-88.

23. Cleton A, Rossenu S, Crauwels H, et al. A single-dose, open-label, parallel, randomized, dose-proportionality study of paliperidone after intramuscular injections of paliperidone palmitate in the deltoid or gluteal muscle in patients with schizophrenia. J Clin Pharmacol. 2014; 54(9):1048-1057.

24. Kramer M, Litman R, Hough D, et al. Paliperidone palmitate, a potential long-acting treatment for patients with schizophrenia. Results of a randomized, double-blind, placebo-controlled efficacy and safety study. Int J Neuropsychopharmacol. 2010;13(5):635-647.

25. Nasrallah HA, Gopal S, Gassmann-Mayer C, et al. A controlled, evidence-based trial of paliperidone palmitate, a long-acting injectable antipsychotic, in schizophrenia. Neuropsychopharmacology. 2010; 35(10):2072-2082. 
26. Gopal S, Hough DW, Xu H, et al. Efficacy and safety of paliperidone palmitate in adult patients with acutely symptomatic schizophrenia: a randomized, double-blind, placebo-controlled, dose-response study. Int Clin Psychopharmacol. 2010;25(5):247-256.

27. Pandina GJ, Lindenmayer JP, Lull J, et al. A randomized, placebocontrolled study to assess the efficacy and safety of 3 doses of paliperidone palmitate in adults with acutely exacerbated schizophrenia. J Clin Psychopharmacol. 2010;30(3):235-244.

28. Alphs L, Bossie CA, Sliwa JK, Ma YW, Turner N. Onset of efficacy with acute long-acting injectable paliperidone palmitate treatment in markedly to severely ill patients with schizophrenia: post hoc analysis of a randomized, double-blind clinical trial. Ann Gen Psychiatry. 2011; 10(1):12.

29. Hough D, Gopal S, Vijapurkar U, Lim P, Morozova M, Eerdekens M. Paliperidone palmitate maintenance treatment in delaying the time-torelapse in patients with schizophrenia: a randomized, double-blind, placebo-controlled study. Schizophr Res. 2010;116(2-3):107-117.

30. Gopal S, Vijapurkar U, Lim P, Morozova M, Eerdekens M, Hough D. A 52-week open-label study of the safety and tolerability of paliperidone palmitate in patients with schizophrenia. J Psychopharmacol. 2011;25(5):685-697.

31. Fu DJ, Turkoz I, Walling D, Lindenmayer JP, Schooler NR, Alphs L. Paliperidone palmitate once-monthly maintains improvement in functioning domains of the Personal and Social Performance scale compared with placebo in subjects with schizoaffective disorder. Schizophr Res. Epub 2017 Apr 25.

32. Fleischhacker WW, Gopal S, Lane R, et al. A randomized trial of paliperidone palmitate and risperidone long-acting injectable in schizophrenia. Int J Neuropsychopharmacol. 2012;15(1):107-118.

33. Pandina G, Lane R, Gopal S, et al. A double-blind study of paliperidone palmitate and risperidone long-acting injectable in adults with schizophrenia. Prog Neuropsychopharmacol Biol Psychiatry. 2011; 35(1):218-226.

34. Fu DJ, Bossie CA, Kern SJ, Ma YW, Alphs L. Paliperidone palmitate versus risperidone long-acting injection in markedly-to-severely ill schizophrenia subjects: onset of efficacy with recommended initiation regimens. Clin Schizophr Relat Psychoses. 2014;8(2): 101-109, 109A.

35. Fu DJ, Bossie CA, Sliwa JK, Ma YW, Alphs L. Paliperidone palmitate versus oral risperidone and risperidone long-acting injection in patients with recently diagnosed schizophrenia: a tolerability and efficacy comparison. Int Clin Psychopharmacol. 2014;29(1):45-55.

36. Li H, Rui Q, Ning X, Xu H, Gu N. A comparative study of paliperidone palmitate and risperidone long-acting injectable therapy in schizophrenia. Prog Neuropsychopharmacol Biol Psychiatry. 2011; 35(4):1002-1008.

37. Koshikawa Y, Takekita Y, Kato M, et al. The comparative effects of risperidone long-acting injection and paliperidone palmitate on social functioning in schizophrenia: a 6-month, open-label, randomized controlled pilot trial. Neuropsychobiology. 2016;73(1):35-42.

38. Takekita Y, Koshikawa Y, Fabbri C, et al. Cognitive function and risperidone long-acting injection vs. paliperidone palmitate in schizophrenia: a 6-month, open-label, randomized, pilot trial. BMC Psychiatry. 2016;16:172.

39. Alphs L, Mao L, Lynn SH, Benson C. A pragmatic analysis comparing once-monthly paliperidone palmitate versus daily oral antipsychotic treatment in patients with schizophrenia. Schizophr Res. 2016; 170(2-3):259-264.

40. Kwon JS, Kim SN, Han J, et al. Satisfaction of immediate or delayed switch to paliperidone palmitate in patients unsatisfied with current oral atypical antipsychotics. Int Clin Psychopharmacol. 2015; 30(6):320-328.

41. Naber D, Hansen K, Forray C, et al. Qualify: a randomized head-to-head study of aripiprazole once-monthly and paliperidone palmitate in the treatment of schizophrenia. Schizophr Res. 2015;168(1-2):498-504.
42. McEvoy JP, Byerly M, Hamer RM, et al. Effectiveness of paliperidone palmitate vs haloperidol decanoate for maintenance treatment of schizophrenia: a randomized clinical trial. JAMA. 2014;311(19): 1978-1987.

43. Rosenheck RA, Leslie DL, Sint KJ, et al. Cost-effectiveness of longacting injectable paliperidone palmitate versus haloperidol decanoate in maintenance treatment of schizophrenia. Psychiatr Serv. 2016;67(10): 1124-1130.

44. Schreiner A, Aadamsoo K, Altamura AC, et al. Paliperidone palmitate versus oral antipsychotics in recently diagnosed schizophrenia. Schizophr Res. 2015;169(1-3):393-399.

45. Gopal S, Xu H, McQuarrie K, et al. Caregiver burden in schizophrenia following paliperidone palmitate long acting injectables treatment: pooled analysis of two double-blind randomized phase three studies. NPJ Schizophr. 2017;3(1):23.

46. Attard A, Olofinjana O, Cornelius V, Curtis V, Taylor D. Paliperidone palmitate long-acting injection - prospective year-long follow-up of use in clinical practice. Acta Psychiatr Scand. 2014;130(1):46-51.

47. Hargarter L, Lahaye M, Cherubin P, et al. Treatment response and tolerability with once-monthly paliperidone palmitate initiated shortly after hospital admission in patients with schizophrenia. World J Biol Psychiatry. Epub 2017 Jun 8.

48. Baser O, Xie L, Pesa J, Durkin M. Healthcare utilization and costs of veterans health administration patients with schizophrenia treated with paliperidone palmitate long-acting injection or oral atypical antipsychotics. J Med Econ. 2015;18(5):357-365.

49. Bressington D, Stock J, Hulbert S, MacInnes D. A retrospective observational study of the effectiveness of paliperidone palmitate on acute inpatient hospitalization rates. Int Clin Psychopharmacol. 2015; 30(4):230-236.

50. Doshi JA, Pettit AR, Stoddard JJ, Zummo J, Marcus SC. Concurrent oral antipsychotic drug use among schizophrenia patients initiated on long-acting injectable antipsychotics post-hospital discharge. J Clin Psychopharmacol. 2015;35(4):442-446.

51. Schreiner A, Bergmans $\mathrm{P}$, Cherubin $\mathrm{P}$, et al. A prospective flexible-dose study of paliperidone palmitate in nonacute but symptomatic patients with schizophrenia previously unsuccessfully treated with oral antipsychotic agents. Clin Ther. 2014;36(10):1372-1388.

52. Schreiner A, Bergmans P, Cherubin P, et al. Paliperidone palmitate in non-acute patients with schizophrenia previously unsuccessfully treated with risperidone long-acting therapy or frequently used conventional depot antipsychotics. J Psychopharmacol. 2015;29(8): 910-922.

53. Hargarter L, Cherubin P, Bergmans P, et al. Intramuscular long-acting paliperidone palmitate in acute patients with schizophrenia unsuccessfully treated with oral antipsychotics. Prog Neuropsychopharmacol Biol Psychiatry. 2015;58:1-7.

54. Zhao J, Li L, Shi J, et al. Safety and efficacy of paliperidone palmitate 1-month formulation in Chinese patients with schizophrenia: a 25-week, open-label, multicenter, Phase IV study. Neuropsychiatr Dis Treat. 2017; 13:2045-2056.

55. Lafeuille MH, Grittner AM, Fortier J, et al. Comparison of rehospitalization rates and associated costs among patients with schizophrenia receiving paliperidone palmitate or oral antipsychotics. Am J Health Syst Pharm. 2015;72(5):378-389.

56. Montalvo I, Ortega L, Lopez X, et al. Changes in prolactin levels and sexual function in young psychotic patients after switching from long-acting injectable risperidone to paliperidone palmitate. Int Clin Psychopharmacol. 2013;28(1):46-49.

57. Morrato EH, Parks J, Campagna EJ, et al. Comparative effectiveness of injectable paliperidone palmitate versus oral atypical antipsychotics: early postmarketing evidence. J Comp Eff Res. 2015;4(2):89-99.

58. Taylor D, Olofinjana O. Long-acting paliperidone palmitate - interim results of an observational study of its effect on hospitalization. Int Clin Psychopharmacol. 2014;29(4):229-234. 
59. Taylor DM, Sparshatt A, O’Hagan M, Dzahini O. Paliperidone palmitate: factors predicting continuation with treatment at 2 years. Eur Neuropsychopharmacol. 2016;26(12):2011-2017.

60. Taylor DM, Sparshatt A, O'Hagan M, Dzahini O. Effect of paliperidone palmitate on hospitalisation in a naturalistic cohort - a four-year mirror image study. Eur Psychiatry. 2016;37:43-48.

61. Voss EA, Ryan PB, Stang PE, Hough D, Alphs L. Switching from risperidone long-acting injectable to paliperidone long-acting injectable or oral antipsychotics: analysis of a Medicaid claims database. Int Clin Psychopharmacol. 2015;30(3):151-157.

62. Whale R, Pereira M, Cuthbert S, Fialho R. Effectiveness and predictors of continuation of paliperidone palmitate long-acting injection treatment: a 12-month naturalistic cohort study. J Clin Psychopharmacol. 2015;35(5):591-595.

63. Zhang F, Si T, Chiou CF, et al. Efficacy, safety, and impact on hospitalizations of paliperidone palmitate in recent-onset schizophrenia. Neuropsychiatr Dis Treat. 2015;11:657-668.

64. Pesa JA, Doshi D, Wang L, Yuce H, Baser O. Health care resource utilization and costs of California Medicaid patients with schizophrenia treated with paliperidone palmitate once monthly or atypical oral antipsychotic treatment. Curr Med Res Opin. 2017;33(4):723-731.

65. Pilon D, Muser E, Lefebvre P, Kamstra R, Emond B, Joshi K. Adherence, healthcare resource utilization and Medicaid spending associated with once-monthly paliperidone palmitate versus oral atypical antipsychotic treatment among adults recently diagnosed with schizophrenia. BMC Psychiatry. 2017;17(1):207.

66. Emsley R, Hargarter L, Bergmans P, et al. Once-monthly paliperidone palmitate in early stage schizophrenia - a retrospective, non-interventional 1-year study of patients with newly diagnosed schizophrenia. Neuropsychiatr Dis Treat. 2017;13:2261-2269.
67. Carpiniello B, Pinna F. Critical appraisal of 3-monthly paliperidone depot injections in the treatment of schizophrenia. Drug Des Devel Ther. 2016;10:1731-1742.

68. Berwaerts J, Liu Y, Gopal S, et al. Efficacy and safety of the 3-month formulation of paliperidone palmitate vs placebo for relapse prevention of schizophrenia: a randomized clinical trial. JAMA Psychiatry. 2015;72(8):830-839.

69. Savitz AJ, Xu H, Gopal S, et al. Efficacy and safety of paliperidone palmitate 3-month formulation for patients with schizophrenia: a randomized, multicenter, double-blind, noninferiority study. Int $J$ Neuropsychopharmacol. 2016;19(7).

70. Savitz AJ, Xu H, Gopal S, Nuamah I, Hough D, Mathews M. Paliperidone palmitate 3-month treatment results in symptomatic remission in patients with schizophrenia: a randomized, multicenter, double-blind, and noninferiority study. Int Clin Psychopharmacol. 2017;32(6):329-336.

71. Savitz AJ, Xu H, Gopal S, et al. Efficacy and safety of paliperidone palmitate three-monthly formulation in East Asian patients with schizophrenia: subgroup analysis of a global, randomized, double-blind, Phase III, noninferiority study. Neuropsychiatr Dis Treat. 2017;13: 2193-2207.

72. Lamb YN, Keating GM. Paliperidone palmitate intramuscular 3-monthly formulation: a review in schizophrenia. Drugs. 2016;76(16): 1559-1566.

73. Weiden PJ, Kim E, Bermak J, Turkoz I, Gopal S, Berwaerts J. Does half-life matter after antipsychotic discontinuation? A relapse comparison in schizophrenia with 3 different formulations of paliperidone. J Clin Psychiatry. 2017;78(7):e813-e820.
Neuropsychiatric Disease and Treatment

\section{Publish your work in this journal}

Neuropsychiatric Disease and Treatment is an international, peerreviewed journal of clinical therapeutics and pharmacology focusing on concise rapid reporting of clinical or pre-clinical studies on a range of neuropsychiatric and neurological disorders. This journal is indexed on PubMed Central, the 'PsycINFO' database and CAS,

\section{Dovepress}

and is the official journal of The International Neuropsychiatric Association (INA). The manuscript management system is completely online and includes a very quick and fair peer-review system, which is all easy to use. Visit http://www.dovepress.com/testimonials.php to read real quotes from published authors. 